\title{
The PIR-International databases
}

\author{
Winona C.Barker, David G.George, Hans-Werner Mewes ${ }^{1}$, Friedhelm Pfeiffer ${ }^{1}$ and \\ Akira Tsugita ${ }^{2}$ \\ Protein Information Resource, National Biomedical Research Foundation, Georgetown University \\ Medical Center, USA, 'Martinsried Institute for Protein Sequences, Max Planck Institute for \\ Biochemistry, Martinsried, Germany and ${ }^{2}$ Japan International Protein Information Database, Science \\ University of Tokyo, Noda, Japan
}

\begin{abstract}
PIR-International is an association of macromolecular sequence data collection centers dedicated to fostering international cooperation as an essential element in the development of scientific databases. PIR-International is most noted for the Protein Sequence Database. This database originated in the early 1960's with the pioneering work of the late Margaret Dayhoff as a research tool for the study of protein evolution and intersequence relationships; it is maintained as a scientific resource, organized by biological concepts, using sequence homology as a guiding principle. PIRInternational also maintains a number of other genomic, protein sequence, and sequence-related databases. The databases of PIR-International are made widely available. This paper briefly describes the architecture of the Protein Sequence Database, a number of other PIR-International databases, and mechanisms for providing access to and for distribution of these databases.
\end{abstract}

\section{PIR-International}

PIR-International is a tripartite association of protein sequence data centers: the Protein Information Resource (PIR) at National Biomedical Research Foundation (NBRF) at Georgetown University, Washington DC, USA, which is directed by $\mathrm{Dr}$ Winona C. Barker; the Martinsried Institute for Protein Sequences (MIPS) at the Max Planck Institute for Biochemistry, Martinsried, Germany, directed by Dr Hans-Werner Mewes; and the Japan International Protein Information Database (JIPID) at the Science University of Tokyo, directed by Professor Akira Tsugita. The unique goals of PIR-International are (1) to dynamically maintain a complete and comprehensive set of published protein sequences and related information in accordance with current biological understanding, (2) to organize these data by similarity and evolutionary relationship, and (3) to add biochemical information that is displayed in a consistent and comprehensive form.

The success of the PIR-International collaboration is seen both in the growth of the database and in the increase in the contributions of MIPS and JIPID. From December 1988 until March 1993, there has been a five-fold increase in the number of entries in the Protein Sequence Database. At the beginning of 1989, the public database totaled 10,527 entries and about 2500 additional sequences were in the unverified backlog. Of these 13,000 entries, at least $95 \%$ had been entered at NBRF. With the establishment of MIPS and JIPID as full-function nodes of PIR-International, more than $50 \%$ of the current data input originates from Asian and European sources.

The protein sequence database

The Protein Sequence Database was initiated in the early 1960's by Margaret O.Dayhoff [1-3] for the express purpose of providing a dataset for the support of research on the interrelationships and evolution of proteins. Supporting such research requires that an up-to-date representation of the scientific understanding of the information be maintained. The central goal of the database project has been to maintain this perspective by merging all sequence data corresponding to a particular protein into one representative structure that is consistently annotated with information describing its genetic origin and expression and its biological function.

Sequences in the Protein Sequence Database are derived from publications in books or scientific journals, or from submission of the sequence to a sequence database. PIR-International stores the sequences as published in a sequence archive. Each entry in the sequence archive is identified by its associated accession number. In addition, the reference information is stored in a reference archive. Each reference in the reference archive is identified by its associated reference number.

As a first step, entered data are added to the sequence and reference archives and become immediately accessible as a source of input to the Protein Sequence Database. Upon addition to the archive, data are compared with the archive to avoid duplication. In case of errors detected in later processing stages, these are corrected in the archive so that an accurate permanent record can be maintained. A preliminary entry contains reference and sequence, a title as a short description of the protein, an accession number, a reference number, and an entry identification code. Whenever applicable, the formal nomenclature for the species and a cross-reference to the nucleotide sequence database is given.

As a second step, the basic information is verified by comparison to the publication. Basic information consists of the protein name, the species name, the type of molecule sequenced, and an indication if fragmentary data are reported. Entries on this level typically contain additional information (e.g., features).

Annotation is complete if all information within the scope of the database is accumulated and data from multiple determinations of the sequence of the same molecule are combined into a single entry. These entries are organized by function and sequence 
similarity. Entries are subject to continuous modifications to reflect the current biological knowledge.

To provide access to sequence data that are as yet unprocessed by PIR-International but available in other sources, a data set compiled by MIPS, which is called PATCHX, is available. The superset of PATCHX and the Protein Sequence Database was formerly called MIPSX. This collection is maintained to allow exhaustive homology searches. The data set is distributed on the CD-ROM (see below) and available separately on magnetic media.

\section{Merging reports of the same sequence}

PIR-International aims to merge all reports of a given sequence into a single entry in which the most up-to-date understanding of the protein structure is presented. Therefore entries are dynamic. For example, subsequent work revising portions of the sequence may result in modifications to the 'canonical' sequence represented in the entry. Moreover, clarification of the original data may result in the separation of information previous thought to refer to the same protein sequence. To enable accurate representation of the sequence reported in each citation and recovery of the individually reported sequences, we have developed a concise syntax for representing differences between reported sequences. For example, 'Residues: 23-69, 'N',71-80' specifies that the sequence in this report can be regenerated from the sequence shown in the entry by taking residues $23-80$ and substituting asparagine for the residue at position 70 . Using the sequence specification, the ATLAS program can regenerate the reported sequence. Software comparisons of merged entries to the corresponding archive entries guarantee that the sequence data do not become corrupted during merging or editing.

\section{The superfamily concept and placement in the database}

A superfamily is a group of proteins that share sequence similarity due to common ancestry [4,5]. From the mid-1970's until recently, the entries in the Protein Sequence Database were organized into superfamilies, families, subfamilies, entries, and subentries by assigning to each entry a set of numbers that uniquely specified both its order (or placement) in the database and its relationship to other entries [6]. This hierarchical organization has proved to be very fruitful and has been employed in a variety of investigations [7-10]. However, this structure was designed before it was recognized that a given sequence can be assigned to more than one superfamily and that similarity among proteins within a superfamily may be restricted to regions or domains. In grouping proteins that share one particular domain, portions of the sequences that are dissimilar or even related to sequences in other superfamilies had to be ignored.

We have addressed these problems by decoupling the concept of superfamily classification from relative placement. Superfamily assignment is based on homologous protein domains (although, in most cases, the domain extends the entire length of the protein). Superfamily relationships are indicated in a Superfamily Record, which has been added to all entries in PIR1 (except hypothetical proteins) and to many entries in PIR2. This record may contain one or more superfamily names. The five-number classification used for placement in PIR1 distinguishes groups of proteins that are homologous over the majority of their lengths. This clustering provides an overview of the types of proteins present in the database and a means of assessing the depth of data available concerning specific groups of proteins. The partitioning of the sequences into more uniform groups is an ongoing process with the most diverse groups being accorded highest priority.

\section{Standardization within and among databases}

PIR-International is vigorously pursuing a multifaceted effort toward standardization of the various types of information represented in our Protein Sequence Database. The general approach is to partition the data into well-defined fields, to subdivide terminology standardization into a series of manageable tasks, and to distribute these tasks among the collaborators. When available, we adhere to terminology standards recommended by international nomenclature commissions. Initially we standardized journal abbreviations (in cooperation with the NLM and the nucleotide sequence databases) [11], species names that appear in the database, and enzyme names (using the names recommended by the Enzyme Nomenclature Commission [12]). The PIR-International taxonomic listing is distributed with the database and is used by a number of other database centers, including GenBank [13], EMBL [14], BioMagResBank [15], the Brookhaven Protein Data Bank [16], and NCBI [17]. These groups share taxonomic information on a regular basis and have jointly developed a network of taxonomic experts for electronic consultation.

During the past year, major efforts were directed toward standardization of keywords, superfamily names, and features. Procedures for enforcing conformance to these standards were developed. The following fields are now checked by software: protein name and Enzyme Commission number for enzymes, species name record, organism name and special genetic code (if used) in entry title, reference number and citation, journal abbreviation, superfamily name, and keywords. Some of these checks include mandatory links between the information in different records (e.g., the organism information in the entry title must be compatible with the formal species name given in the species name record). These checks are regularly executed on the entire dataset and are always applied whenever an entry has been significantly modified.

Results of the keyword standardization project can be seen by a comparison of Release 30 (September 1991) and Release 34 (September 1992) with respect to how many times each keyword appeared in the database. The number of different keywords used was reduced from 1614 to 1037 , with the bulk of the reduction being for keywords or variants that appeared infrequently. At the same time, the number of keywords that appear more than 50 times in the database doubled. In 1992, we began the standardization of feature annotations. Fields recording active sites, binding sites, modified sites, and disulfide (and other) bonds have been largely standardized. Several of the database centers represented at the 1992 meeting of the CODATA Task Group on Macromolecules indicated a need for guidelines for representing modified amino acids. With cooperation from scientists at Chemical Abstracts, NBRF has compiled a database of types of amino acid residue modifications and made it available for criticism or use by other databases.

\section{The alignment database}

NBRF has developed a system for the construction, storage, and retrieval of alignments of protein sequences. In order to have meaningful alignments, to avoid complications, and to ensure easy handling and display, groups of at least three sequences of comparable lengths and less than 55\% different were chosen from the PIR1 section of the PIR-International Protein Sequence Database for the initial alignment data set. Alignments were restricted to single-domain or multidomain proteins that share a common domain composition, order, and structure. For families with a large number of sequences, an alignment containing 
representative sequences only was constructed. Also displayed are a list of the codes and titles of the sequences in each alignment and a matrix showing the percentage difference and the number of differences between each pair of aligned sequences. As of Release 4.0 (March 1993), the Alignment Database contained 956 entries.

\section{The NRL_3D sequence-structure database}

NBRF prepares regular releases of the NRL_3D SequenceStructure Database [18], derived from sequence information extracted from the Brookhaven Protein Data Bank (PDB) of crystallographic structures. These are restructured to be compatible with PIR software, which has been extended to access this information and to handle transparently the numbering system conversions. The PSQ retrieval program was modified so that the output file for segments matching a test segment may be used to generate input directly from the PDB coordinate files to molecular modeling and graphics programs. This allows the corresponding substructures to be displayed and manipulated. A fully automated conversion procedure, PIR2PDB [19-20], includes routines to detect, report, and work around inconsistencies with PDB format files. Release 12.0 of the NRL_3D Database (March 1993) contained 1630 entries.

\section{Escherichia coli K12 genomic database}

Scientists at JIPID and NBRF collected genomic sequence data from Escherichia coli and developed a model genomic sequence database [21]. This database contains sequence information from the GenBank, EMBL, and NBRF nucleic acid sequence databases, plus information entered directly from published reports. Protein coding regions are annotated in the feature tables, as are additional features such as promoter regions, ShineDalgarno sequences, and transcription termination sequences. The protein coding regions are directly cross-referenced to the PIRInternational Protein Sequence Database and features are formatted to allow direct translation by computer. Overlapping sequences are merged and ordered by map position. When their orientation is known, sequence segments are represented in the same direction (the plus strand). Genetic map positions [22] were directly correlated with the Kohara physical map [23] using an algorithm developed by Kunisawa and coworkers [24]. This algorithm compares restriction fragment lengths and thus directly incorporates information on restriction site distances while avoiding site inversion problems.

\section{Network access to PIR-International and electronic data distribution}

PIR-International data are accessible through electronic data networks on a number of file-servers and FTP sites. NBRF and MIPS operate full-function network fileservers that handle database queries, sequence searches, and sequence submissions, in addition to fileserver requests.

The NBRF network request server responds to 21 database query commands and 7 general service commands. Most of the database query commands are implemented with calls to the ATLAS program. These commands provide simultaneous access to the PIR1, PIR2, PIR3, NRL_3D, PATCHX, Alignment, GenBank, GBNEW, and EMBL databases. Another database query command, SEARCH, is implemented through a version of the FASTA program [25] with output routines modified for network transmission. This command also performs another, unique function: nucleotide sequences are translated in six reading frames and each polypeptide translation is submitted to a FASTA search against the protein sequence databases. The TAXONOMY command uses a special purpose program. HELP instructions are available for all the server commands and for the following topics: Custom Services, Databases, FTP, Gateway_Access, Help_en_Espanol, Help_en_Francais, Hints, IBMVM BITNET, On-Line_Access, PIR_Distribution, VAXVMS_BITNET. Complete instructions for the NBRF fileserver can be obtained by sending an E-mail message containing the command HELP (in the body of the message, not on the Subject line) to FILESERV@GUNBRF.BITNET.

The Protein Sequence Database and all other major sequence databases are also accessible at MIPS by electronic mail query and on-line connection. MIPS forwards database transactions to a propagating network of a number of nodes in Europe to provide most up-to-date information. File servers for sequence searching and for database retrieval are also available (contact mips@ehpmic.mips.biochem.dbp.de for further information).

Through the kind efforts of Bill Pearson at the University of Virginia and Dan Davison at the University of Houston, each PIR-International release and the accompanying NRL_3D release are available for anonymous FTP, WAIS, and Gopher access from the University of Houston Gene-Server. All files are stored as Unix 16-bit compressed files. An ASCII directory contains the CODATA format files and a VMS directory contains the database files and indexes in VMS format. Utilities to uncompress the data are available at that site for non-Unix systems.

\section{The atlas of protein and genomic sequences CD-ROM}

The first Atlas of Protein and Genomic Sequences CD-ROM was released in July 1992. Beginning with Release 34 (September 1992) the CD-ROM has been released quarterly. The CD-ROM contains the ATLAS Information Retrieval System, the FASTA program for similarity searches, the PIR-International Protein Sequence Database, the GenBank database, the NRL_3D database, the Alignment Database, the PATCHX database, and the Escherichia coli K12 Genomic Database.

The ATLAS program is a fully integrated multidatabase access program that allows simultaneous access to multiple databases. Although designed primarily to handle macromolecular sequence databases, it can operate on textual databases. The program employs a single multidatabase, multifield index structure. This design provides a framework that allows simultaneous retrieval from any selected set of databases and any combination of fields within those databases. With each release of the CD-ROM, the 'Atlas of Protein and Genomic Sequences Installation and User's Guide' is updated. Although included on the CD-ROM, it can also obtained separately in printed form.

The CD-ROM is formatted in accordance with the ISO 9660 standard and can be read from any computer system supporting this standard. The ATLAS program currently runs on PC-DOS, VAX/VMS, OpenVMS, and ULTRIX systems; versions for SunOS, Silicon Graphics, various other UNIX-based systems, and Macintosh computers are being developed. The program is written entirely in the $\mathrm{C}$ computer language and complies with the ANSI standard.

\section{Data distribution on magnetic tapes}

The PIR-International Protein Sequence Database is distributed four times yearly on magnetic media in VAX/VMS and ASCII formats. The data and programs are available on 9-track tape, on TK50 and TK70 streaming tape cartridges, and on DAT 4mm cartridges. VMS users have the option of VAX/VMS copy or 
backup formats. The tapes currently include four protein sequence datasets (PIR1, PIR2, PIR3, and NRL_3D); files of associated information such as taxonomic classification of organisms, special genetic codes, list of superfamilies; update information; extensive indexes; and documentation. In addition, the VAX/VMS tape includes the PSQ and NAQ retrieval systems, software to create databases that can be accessed by PSQ or NAQ from usersupplied sequence entries or from GenBank or EMBL databases, software to create the ASCII flat-file version of the database from the VAX/VMS version, and indexes to make the Protein Sequence Database compatible with the GCG software package. ASCII card image format tapes do not include retrieval software; however, files are supplied containing indexes to authors, accession numbers, reference numbers, species, superfamily names, citations, keywords, and features. Tapes in both formats contain documentation as well as additional files containing the species names (ordered in a taxonomic hierarchy), journal abbreviations, and special genetic codes used in the database.

The PIR-International Protein Sequence Database is distributed by many other vendors in conjunction with software packages. The nodes of PIR-International reserve any rights on their intellectual properties and are not responsible for the versions of the database supplied by any secondary sources. Although users may find these software-data packages convenient, they should be aware that the database supplied may not be the latest release and may not include all of the information available in the original.

How to obtain PIR-International databases, software, and newsletters

For information on currently available database releases, or other services, or for a copy of the PIR Newsletter, contact the PIR Technical Services Coordinator, National Biomedical Research Foundation, 3900 Reservoir Road, NW, Washington, D.C. 20007; telephone +1 202 687-2121; FAX +1 202 687-1662; electronic mail PIRMAIL@nbrf.georgetown.edu. In Europe, contact MIPS: Martinsrieder Institut für Proteinsequenzen, MaxPlanck-Institut für Biochemie, D-8033 Martinsried bei München, FRG; telephone +49 898578 2656; FAX +49 898578 2655; electronic mail mips@ehpmic.mips.biochem.dbp.de. In Asia or Australia, please contact JIPID: Japan International Protein Information Database, Science University of Tokyo, 2641 Yamazaki, Noda 278, Japan; telephone +81 471 239778; FAX +81 471 221544; electronic mail TSUGITA@JPNSUT31.BITNET and EX5292@JPNSUT30.BITNET.

\section{ACKNOWLEDGEMENTS}

The Protein Identification Resource is supported by National Institutes of Health Grant LM05206 and National Science Foundation Grant DIR9107540. Development of the ATLAS CDROM was partially supported by a grant from Digital Equipment Corporation. MIPS is supported by the Max-Planck-Gesellschaft, the Forschungszentrum f. Umwelt und Gesundheit (GSF) and the European Economic Community BRIDGE Programme Grants BIOT-CT-0167 and 0172.

\section{REFERENCES}

1. Dayhoff, M.O., Eck, R.V., Chang, M.A., and Sochard, M.R. (1965) Atlas of Protein Sequence and Structure. National Biomedical Research Foundation, Silver Spring, MD.
2. Dayhoff, M.O. (1972) Atlas of Protein Sequence and Structure vol. 5. National Biomedical Research Foundation, Washington, DC.

3. Dayhoff, M.O. (1979) Atlas of Protein Sequence and Structure vol. 5, Supplement 3. National Biomedical Research Foundation, Washington, DC.

4. Dayhoff, M.O., McLaughlin, P.J., Barker, W.C., and Hunt, L.T. (1975) Naturwissenschaften 62, 154-161.

5. Dayhoff, M.O. (1976) Fed. Proc. 35, 2132-2138.

6. Barker, W.C., George, D.G., Hunt, L.T., and Garavelli, J.S. (1991) Nucleic Acids Res. 19, 2231-2236.

7. Pearson, W.R. (1991) Genomics 11, 635-650.

8. Seto, Y., Ikeuchi, Y., and Kanehisa, M. (1990) PROTEINS: Struct. Funct. Genet. 8, 341-351.

9. Lipman, D.J., and Pearson, W.R. (1985) Science 227, 1435-1441.

10. Guigo, R., Johansson, A., and Smith, T. (1991) Comput. Appl. Biosci. 7, 309-315.

11. National Biomedical Research Foundation (1989) PIR Newsletter, No. 7.

12. Nomenclature Committee of the International Union of Biochemistry (1992) Enzyme Nomenclature. Academic Press, Orlando, FL.

13. Burks, C., Cinkosky, M.J., Fischer, W.M., Gilna, P., Hayden, J. E.-D., Keen, G.M., Kelly, M., Kristofferson, D., and Lawrence, J. (1992) Nucleic Acids Res. 20, 2065-2069.

14. Higgins, D.G., Fuchs, R., Stoehr, P.J., and Cameron, G.N. (1992) Nucleic Acids Res. 20, 2071-2074.

15. Markley, J.L., Seavey, B.R., and Farr, E. (1991) CODATA Bull. 23(4), 85-88.

16. Abola, E.E., Bernstein, F.C., Bryant, S.H., Koetzle, T.F., and Weng, J. (1987) Protein data bank, pp. 107-132 in Crystallographic DatabasesInformation Content, Software Systems, Scientific Applications, F.H. Allen, G. Bergerhoff, and R. Sievers, eds. Data Commission of the International Union of Crystallography, Cambridge.

17. Benson, D. (1991) CODATA Bull. 23(4), 76-78.

18. Pattabiraman, N., Namboodiri, K., Lowrey, A., and Gaber, B.P. (1990) Protein Seq. Data Anal. 3, 387-405.

19. Kusunoki, M., George, D.G., Namboodiri, K., and Pattabiraman, N. (1991) Protein Seq. Data Anal. 4, 333-336.

20. National Biomedical Research Foundation (1992) PIR Newsletter, No. 12.

21. Kunisawa, T., Nakamura, M., Watanabe, H., Otsuka, J., Tsugita, A., Yeh, L.-S.L., George, D.G., and Barker, W.C. (1990) Protein Seq. Data Anal. 3, 157-162.

22. Bachmann, B.J. (1983) Microbiol. Rev. 47, 180-230.

23. Kohara, Y., Akiyama, K., and Isono, K. (1987) Cell 50, 495-508.

24. Watanabe, H., and Kunisawa, T. (1990) Protein Seq. Data Anal. 3, 149-156.

25. Pearson, W.R., and Lipman, D.J. (1988) Proc. Nat. Acad. Sci. USA 85 , 2444-2448. 